\title{
Iniciação Científica por Meio da Pesquisa Experimental In Vivo: Relato de Experiência Acadêmica
}

\author{
Genésio Lopes Mercês de Almeida ${ }^{1}$, Fernanda Novaes de Sousa ${ }^{2}$, Camilla Leandro de Freitas ${ }^{1}$, \\ Laís Silvi Massulo Ribeiro ${ }^{1}$, Rayane dos Santos Ferreira ${ }^{3}$, Andreia Aparecida Barbosa ${ }^{2}$, Bruno \\ Anjos Blanco ${ }^{1}$, André Luiz Thomaz de Souza ${ }^{3 *}$
}

${ }^{1}$ Departamento de Farmácia das Faculdades Integradas do Vale do Ribeira

${ }^{2}$ Departamento de Biologia das Faculdades Integradas do Vale do Ribeira

${ }^{3}$ Departamento de Enfermagem das Faculdades Integradas do Vale do Ribeira

* Autor para correspondência: alfenas2@hotmail.com

\section{RESUMO}

A pesquisa científica é o alicerce para a evolução do conhecimento humano. Este estudo tem como objetivo descrever a experiência acadêmica na iniciação científica por meio da pesquisa experimental in vivo. A iniciação científica foi desenvolvida com o uso de terapias alternativas no tratamento da cicatrização da pele em ratos. O público-alvo envolveu alunos dos cursos de bacharelado em Enfermagem, Farmácia e Biologia. Durante a iniciação científica, os alunos participaram da leitura crítica de artigos científicos, da redação do projeto de pesquisa, do desenvolvimento experimental, de protocolos experimentais e de eventos científicos. A experiência resultou para o aluno no avanço na fronteira do conhecimento sobre as ciências básicas e no uso de modelos animais na pesquisa experimental. Contudo, a obtenção de recursos financeiros e humanos representou um obstáculo durante a condução das atividades. Conclui-se que a iniciação científica agrega valores essenciais na formação acadêmica, principalmente no que se refere à capacidade de tomada de decisão e de raciocínio crítico. Além disso, para o contexto deste relato resultou no maior interesse e na procura pela pesquisa científica.

Palavras-Chave: Experimentação Animal; Pesquisa Básica; Ensino.

\begin{abstract}
Scientific research is the foundation for the evolution of human knowledge. This study aims to describe the academic experience in scientific initiation through experimental research in vivo. The scientific initiation was developed with the use of alternative therapies in the treatment in the healing of the skin in rats. The target audience involved students in the bachelor's degree in Nursing, Pharmacy and Biology. During the scientific initiation the students participated in the critical reading of scientific articles, writing research project, experimental development, experimental protocols and scientific events. The experiment resulted in the student advancing the frontier of knowledge about the basic sciences and the use of animal models in experimental research. However, obtaining financial and human resources was an obstacle during the conduct of the activities. It is concluded that the scientific initiation adds essential values in the academic formation, mainly in what refers to the capacity in the decision making and the critical reasoning. In addition, this initiative has resulted in greater interest and demand for scientific research.
\end{abstract}

Keywords: Animal Experimentation; Basic Research; Teaching.

\section{Introdução}

O papel da pesquisa científica essencialmente está vinculado à evolução do conhecimento humano. Sua utilização envolve o uso de ferramentas que buscam investigar, descrever e observar as causas e os efeitos dos fenômenos que cercam a humanidade (PITHON, 2013). Além disso, a pesquisa melhora a qualidade de vida do homem. O desenvolvimento de estudos clínicos e consequentemente a tomada de decisão no contexto profissional são fundamentados no desenvolvimento prévio de estudos in vivo, in 
vitro ou de estudos epidemiológicos (LAUZ et al., 2008).

A pesquisa científica com o uso de modelos experimentais pode ser definida como a materialização parcial de situações reais (FERREIRA et al., 2005). Ela deve ser precisa e demonstrar limitações em relação àquilo que se deseja reproduzir (FERREIRA \& FERREIRA, 2003). A utilização de modelos experimentais é importante nas ciências da saúde, pois auxilia a compreensão sobre os mecanismos das doenças, a ação de fármacos e sobre o funcionamento do organismo. Os modelos experimentais mais utilizados nas ciências da saúde envolvem a cultura de células e tecidos (pesquisa in vitro) e o uso de animais de laboratório (pesquisa in vivo) (FERREIRA et al., 2005).

Os programas de pós-graduação stricto sensu, principalmente das universidades públicas brasileiras, se destacam na produção científica envolvendo a pesquisa in vivo e in vitro; além disso, paralelamente, inserem os alunos na iniciação científica. No entanto, quando se trata do ensino superior particular, em sua maioria, o envolvimento com as atividades de pesquisa ainda é discreto, principalmente as relacionadas com a pesquisa experimental.

No Brasil, a formação universitária no ensino da graduação, especificamente nos cursos das ciências da saúde, é regulamentada por meio das Diretrizes Curriculares Nacionais, as quais determinam que a pesquisa científica é elemento essencial na formação universitária (BRASIL, 2001). Nesse contexto, as instituições de ensino superior devem fomentar condições para que a pesquisa científica seja parte integrante da formação profissional.

Estudos experimentais in vivo com a participação de alunos de graduação se destacam no meio científico, dentre eles a investigação sobre os efeitos do consumo crônico de etanol na biomecânica e no reparo ósseo (HORVATH et al., 2011; GARCIA et al., 2015), os efeitos do toque terapêutico na aceleração da cicatrização da pele (SOUZA et al., 2017) e os efeitos do própolis na cicatrização da pele (PAIXÃO et al., 2014).

Fundamentado no contexto que concebe a iniciação científica inserida na formação universitária e, consequentemente, a necessidade de ampliar o acesso a ela no ensino superior, este estudo tem como objetivo descrever a experiência acadêmica de iniciação científica por meio da pesquisa experimental in vivo.

\section{Método}

Este relato aborda uma experiência sobre a participação acadêmica no desenvolvimento da pesquisa experimental com o uso de modelo animal, envolvendo o tratamento da cicatrização da pele em ratos da linhagem Wistar por meio de terapias alternativas.

\section{Contexto Local}

As Faculdades Integradas do Vale do Ribeira (FVR), local que envolveu este relato de experiência, estão localizadas na microrregião de Registro, São Paulo, Brasil, e atendem doze municípios no seu entorno. As FVR constituem uma instituição de ensino superior particular que conta com cursos distribuídos nas Ciências Exatas, Humanas e Biológicas. A instituição disponibiliza um biotério para a realização de atividades de pesquisa experimental com ratos da linhagem Wistar.

A pesquisa experimental ainda é incipiente nas atividades das FVR. Por se tratar de um método de pesquisa que requer uma série de habilidades, dentre elas destreza no manejo animal, conhecimento das ciências básicas e análises estatísticas, exige do corpo docente e, consequentemente, do aluno, motivação/incentivo para que as atividades ocorram. Esse contexto evidencia o desafio de tornar a pesquisa experimental uma prática comum em nossas atividades.

Com a meta de inserir os alunos dos cursos das ciências da saúde na pesquisa experimental, a instituição conta com o programa de Iniciação Científica Voluntária, o qual possibilita aos acadêmicos serem escolhidos por meio de processo seletivo conforme normas do regimento interno. Participam da iniciação científica envolvendo pesquisa experimental alunos dos cursos de Biologia, Enfermagem e Farmácia. 
As atividades relacionadas à iniciação científica estão atreladas ao desenvolvimento de estudos sobre o uso de terapias alternativas (fitoterápicos e terapias energéticas) no tratamento da cicatrização da pele em ratos da linhagem Wistar. Os alunos são estimulados a trabalhar de forma interdisciplinar, para que ocorra a agregação do conhecimento das diferentes áreas de formação.

\section{Desenvolvimento}

A pesquisa experimental in vivo nas FVR é concebida anualmente nos cursos das ciências da saúde. Na fase inicial, após a seleção dos discentes, são executadas reuniões quinzenais com o objetivo de discutir e aprimorar temas vinculados à construção científica. Nesse contexto, os alunos em conjunto com os docentes produzem projetos de pesquisa sobre os efeitos de terapias alternativas na cicatrização da pele por segunda intenção em ratos da linhagem Wistar, tema este da linha de pesquisa do grupo que conduz a experimentação.

Toda experiência está atrelada à leitura crítica de artigos científicos, ao uso de bases de dados e aos preceitos éticos que norteiam o uso de animais. Além disso, conforme o cronograma estabelecido pelo grupo, os alunos também participam de aprimoramento técnico no manejo animal e no estudo sobre protocolos de pesquisa (Figura 1).

Concluída a etapa da construção e do desenvolvimento do projeto de pesquisa, os alunos são convidados à participação em eventos científicos (Figura 2) e à redação de manuscritos com meta de publicação (SOUZA et al., 2017). Ao término de todas as etapas, ocorre a entrega dos relatórios de iniciação científica e se dá a finalização das atividades.

A partir das experiências vivenciadas pelos acadêmicos em conjunto com os docentes, este relato de experiência contextualiza informações relevantes sobre o desenvolvimento da pesquisa experimental na graduação, o que permite a reflexão sobre o papel deste método de pesquisa na formação universitária. É importante destacar que o desenvolvimento da pesquisa experimental pode oferecer contribuições significativas em diferentes aplicações, resultando na melhora da interseção entre as ciências básicas e o contexto clínico (GÁRNIO, 2011).

Cabe ressaltar as dificuldades que são encontradas para a implementação da iniciação científica, o que envolve a disponibilidade limitada de recursos financeiros e humanos. Contudo, no geral, a iniciação científica por meio da pesquisa experimental in vivo permite ao discente ampliar o horizonte de conhecimento sobre as ciências básicas e o papel da ciência na construção do conhecimento acadêmico.

Um fato que nos chama a atenção é que, após a divulgação institucional sobre os resultados obtidos, a procura e o interesse dos alunos pela iniciação científica aumentaram. Aliado a isso, podemos inferir que a pesquisa na graduação agrega valores essenciais à formação profissional, tais como o raciocínio lógico e a educação permanente.

A pesquisa básica nas diferentes áreas, exatas, humanas e biológicas, representa o alicerce para a formação, a prática profissional e a pesquisa aplicada (GÁRNIO, 2011). Os laboratórios de pesquisa experimental contribuem significativamente na formação de profissionais, o que envolve recursos humanos tanto na graduação quanto na pós-graduação (BASTOS, 2013).

As ciências básicas representam um conjunto de disciplinas enraizadas no modelo biomédico de formação, como fisiologia, anatomia, genética, patologia, microbiologia, dentre outras (BASTOS, 2013). No que tange à pesquisa básica, sua aplicação é fundamental para responder aos desafios da humanidade e consequentemente explicar as manifestações clínicas desencadeadas por muitas doenças. Um exemplo disso está associado ao mapeamento do genoma humano e a sua utilidade no rastreamento de doenças genéticas (ALVES et al., 2004).

No Brasil, a pesquisa experimental em algumas áreas ainda é incipiente, como, por exemplo, na enfermagem. Isso pode ser explicado devido ao pouco conhecimento do enfermeiro em relação à utilização da pesquisa experimental e à sua 

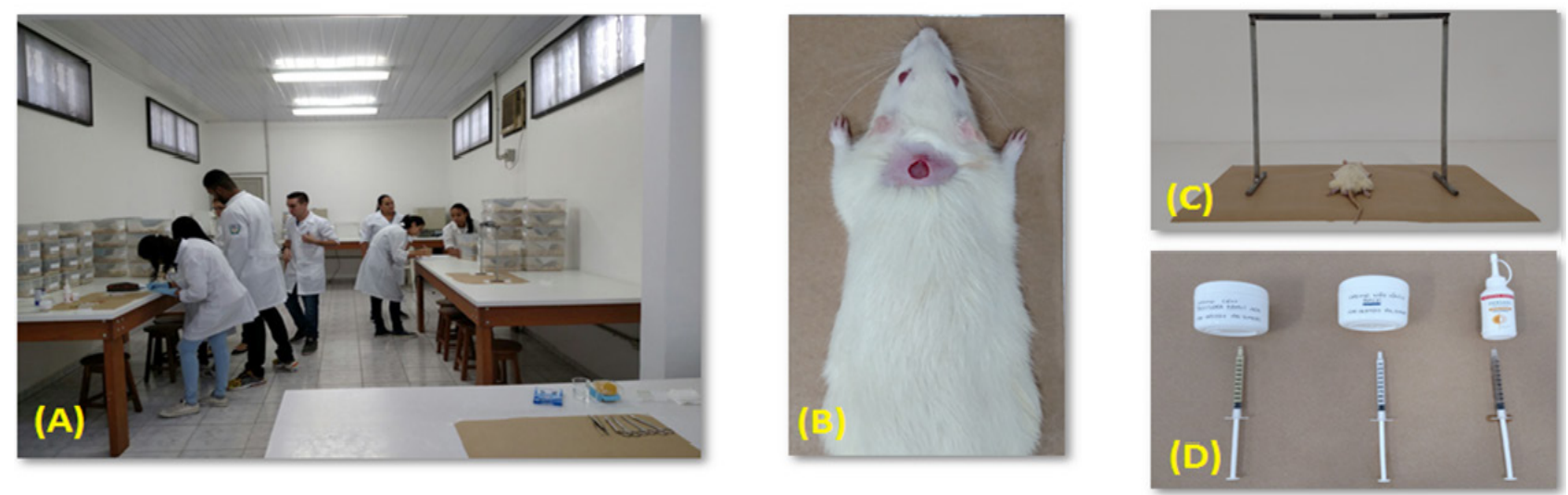

Figura 1 - Rotina de atividades envolvendo a pesquisa experimental com protocolo de cicatrização da pele. (A) Manejo animal; (B) Lesão por segunda intenção; (C) Padronização para registro fotográfico; (D) Tratamento com fitoterápico e controle positivo. Registro, São Paulo, Brasil, 2017.
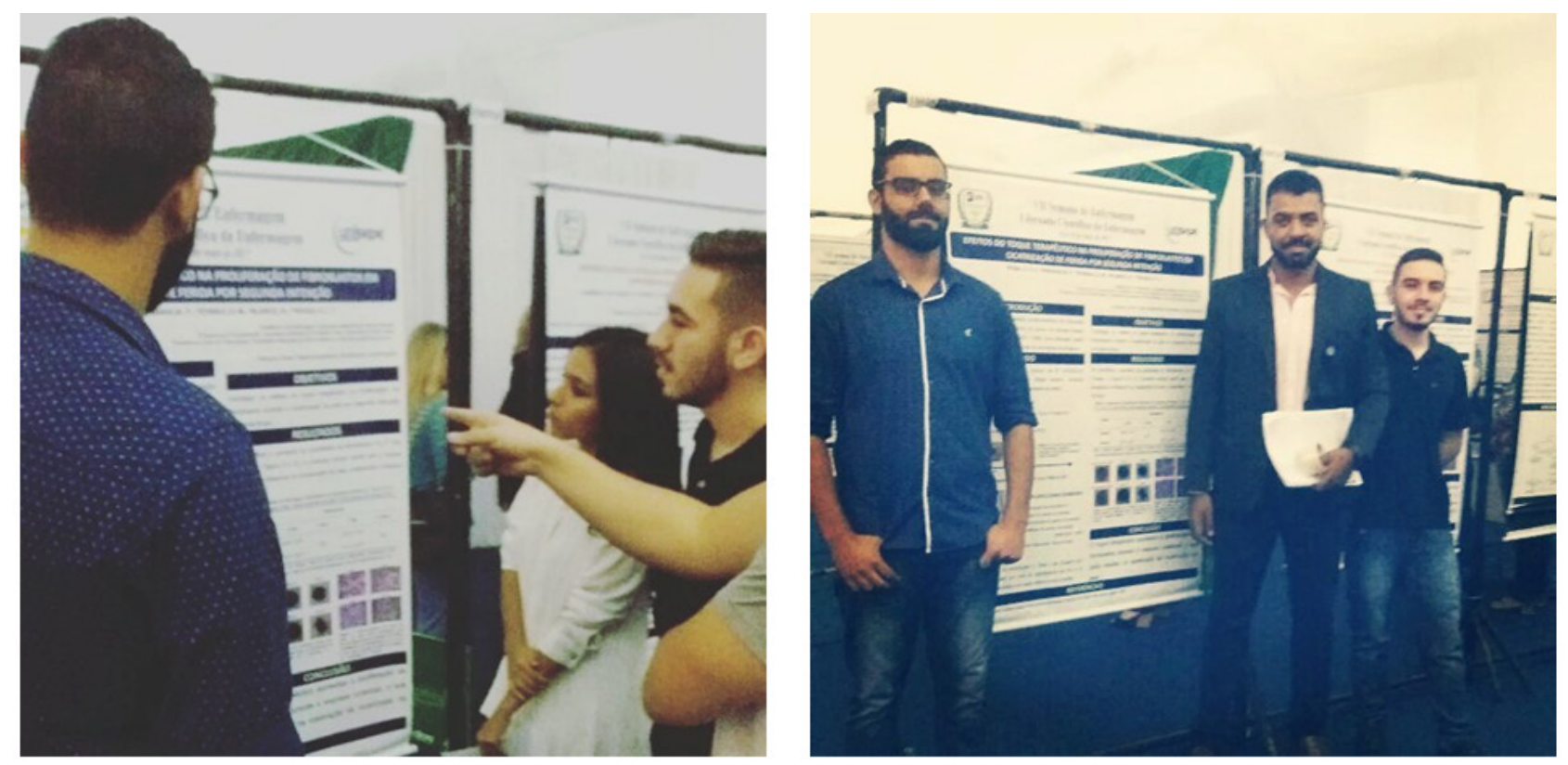

Figura 2 - Participação e divulgação de resultados em evento científico. Registro, São Paulo, Brasil, 2017.

aplicabilidade ao contexto clínico (ALVES et al., 2004; CÁRNIO, 2011). Nesse contexto, destacase a importância do trabalho interdisciplinar no desenvolvimento de estudos que possam subsidiar evidências para a pesquisa clínica, como, por exemplo, a utilização das terapias alternativas (BASTOS et al., 2017).

Considerando os benefícios vinculados à experiência no desenvolvimento da pesquisa experimental vivenciada pelos autores deste estudo, é importante salientar que o trabalho com as ciências básicas devem ser aplicadas em todas as áreas e não exclusivamente na área médica (ALVES et al., 2004). Desse modo, o desafio de ampliar o acesso acadêmico à pesquisa básica permanece, principalmente quando se trata da interdisciplinaridade e do ensino superior particular.

\section{Conclusão}

A experiência acadêmica no desenvolvimento da pesquisa experimental nos conduz à conclusão de que ela deve fazer parte da formação universitária. Esse método de pesquisa possibilita ao aluno de graduação aprofundar o conhecimento das ciências básicas, o que reflete na melhora da formação voltada para o contexto clínico. Cabe destacar que a pesquisa experimental também auxilia o desenvolvimento do raciocínio crítico 
e reflexivo do aluno. Assim, a iniciação científica fortalece o ensino na graduação, visto que representa um espaço de possibilidades para o aprimoramento intelectual, o que contribui para a formação de profissionais qualificados, com rigor científico.

\section{Referências Bibliográficas}

ALVES, Leila M. M.; NOGUEIRA, Maria S.; GODOY, Simone \& CÁRNIO, Evelin C. "Pesquisa Básica na Enfermagem". Rev Latino-Am Enfermagem, vol. 12, n. 1, 2004, pp. 122-127. Disponível em: $<$ http://www.scielo.br/pdf/rlae/v12n1/v12n1a17.pdf>. Acessado em 3 jul. 2017.

BASTOS, Maria L. A. "Pesquisa Básica Experimental em Enfermagem". Rev Enferm UFPE on-line, vol. 7, n. 3, 2013. Disponível em: <http://www. revista.ufpe.br/revistaenfermagem/index.php/revista/ article/view/4663/pdf_2063>. Acessado em 6 jul. 2017.

BRASIL. "Parecer CNE/CES 1133/2001. Diretrizes Curriculares Nacionais dos Gursos de Graduação em Enfermagem, Medicina e Nutrição". Diário Oficial da União. Brasilia, Seção 1E, 7 ago. 2001, p. 131.

CÁRNIO, Evelin C. "Basic Sciences and Nursing". Rev. Latino-Am. Enfermagem, vol. 19, n. 5, set/out. 2011, pp. 10161-62. Disponível em: বhttp:// www.scielo.br/scielo.php? script=sci_arttext\&pi$\mathrm{d}=$ S0104-11692011000500001>. Acessado em 5 jul. 2017.

FERREIRA, Lydia M. \& FERREIRA, Luiz R. K. "Experimental Model: Historic and Conceptual Revision". Acta Cir. Bras., vol. 18, n. spe, 2003, pp. 1-3. Disponível em: <http:// www.scielo.br/scielo.php?script $=$ sci_arttext\&pi$\mathrm{d}=\mathrm{S} 0102-86502003001100001 \& \operatorname{lng}=\mathrm{en} \& \mathrm{nrm}=\mathrm{iso}>$. Acessado em 5 jul. 2017.

; HOCHMAN, Bernardo \& BARBOSA, M. V. J. "Modelos Experimentais em Pesquisa". Acta Cir. Bras., vol. 20, supl. 2, 2005, pp. 28-34. Disponível em: $<$ http://www.scielo.br/scielo.php?script=sci_arttex$\mathrm{t} \&$ pid $=$ S0102-86502005000800008\&lng $=e n \& n r m=i-$ so $>$. Acessado em 4 jul. 2017.

GARCIA, José Antônio D.; SOUZA, André L. T.; CRUZ, Luciana H. C.; MARQUES, Petrus P.; CAMILLI, José Angelo; NAKAGAKI, Wilson R.; ESTEVES, Alessandra; ROSSI-JUNIOR, Wagner C.; FERNANDES, Geraldo J. M.; GUERRA, Flávia D.;
SOARES, Evelise A. "Effects of Ethanol Consumption and Alcohol Detoxification on the Biomechanics and Morphology the Bone in Rat Femurs". Braz. J. Biol., vol. 75, n. 4, 2015, pp. 983-988. Disponível em: $<$ http://www.scielo.br/scielo.php?script=sci_arttext\&pid=S1519-69842015000600983\&lng=en\&nrm=iso $>$. Acessado em 4 jul. 2017.

HORVATH, Renato O.; SILVA, Thiago D.; CALIL-NETO, Jamil; NAKAGAKI, Wilson R.; GARCIA, José Antônio D.; SOARES, Evelise A. "Efeitos do Alcoolismo e da Desintoxicação Alcoólica sobre o Reparo e Biomecânica Óssea”. Acta ortop. Bras., vol. 19, n. 5, 2011, pp. 305-308. Disponível em: $<$ http://www.scielo.br/scielo.php?script=sci_arttext\&pid=S1413-78522011000500008\&lng=en\&nrm=iso>. Acessado em 6 jul. 2017.

LAUZ, Susi.; TELMO, Paula L.; AGUIAR, Patrícia S.; ALMEIDA, Guilherme.; SILVA, Pedro E. A. \& SCAINI, Carlos J. "A Importância dos Biotérios na Pesquisa Experimental". Vittalle, Rio Grande, vol. 20, n. 1, 2008, pp. 25-29. Disponível em: <http:// repositorio.furg.br/bitstream/handle/1/2592/A $\% 20$ import $\%$ c3\%a2ncia $\% 20 \mathrm{de} \% 20$ biot $\%$ c3\%a 9 rios $\% 20$ na $\% 20$ pesquisa $\% 20$ experimental.pdf? sequence $=1>$. Acessado em 4 jul. 2017.

PAIXÃO, Daniella R.; FLAUSINO, Pâmella A.; REIS, Nayara G.; LIMA, G. C.; BERNARDES, Maria T. C. P.; SANTOS, Leandro; GARCIA, José Antônio D. "Efeitos da Própolis na Proliferação de Fibroblastos em Lesões Cutâneas de Rato". Rev Ciênc Farm Básica Apl., vol. 35, n. 3, 2014, pp. 413-417. Disponível em: <http://serv-bib.fcfar.unesp.br/seer/ index.php/Cien_Farm/article/viewFile/2845/2845>. Acessado em 4 jul. 2017.

PITHON, Matheus M. "Importance of the Control Group in Scientific Research". Dental Press J. Orthod., vol. 18, n. 6, 2013, pp. 13-14. Disponível em: 〈http:// www.scielo.br/scielo.php?script $=$ sci_arttext\&pi$\mathrm{d}=$ S2176-94512013000600003\&lng $=e n \& n r m=i$ so $>$. Acessado em: 5 jul. 2017.

SOUZA, André L. T.; ROSA, David. P. C.; BLANCO, Bruno. A.; PASSAGLIA Patrícia \& STABILE, Angelita M. "Effects of Therapeutic Touch on Healing of the Skin in Rats". Explore: The Journal of Science and Healing, vol. 13, n. 5, 2017, pp. 333-338. Disponível em: <http://dx.doi.org/10.1016/j.explore.2017.06.006>. Acessado em 4 jul. 2017. 